\title{
Effect of pulmonary rehabilitation on muscle remodelling in cachectic patients
} with COPD

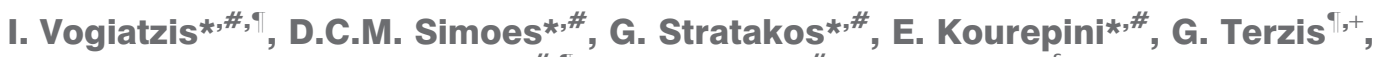 \\ P. Manta ${ }^{+}$, D. Athanasopoulos*,\#, , C. Roussos*,\# , P.D. Wagner ${ }^{\S}$ and S. Zakynthinos*,\#
}

ABSTRACT: It is known that non-cachectic patients with chronic obstructive pulmonary disease (COPD) respond well to pulmonary rehabilitation, but whether cachectic COPD patients are capable of adaptive responses is both important and unknown.

10 cachectic and 19 non-cachectic COPD patients undertook high-intensity cycling training, at the same relative intensity, for 45 min $\cdot$ day $^{-1}, 3$ days $\cdot$ week $^{-1}$ for 10 weeks. Before and after rehabilitation vastus lateralis muscle biopsies were analysed morphologically and for the expression of muscle remodelling factors (insulin-like growth factor (IGF)-I, myogenic differentiation factor D (MyoD), tumour necrosis factor (TNF)- $\alpha$, nuclear factor (NF)-кB and myostatin) and key components of ubiquitin-mediated proteolytic systems (muscle ring finger protein (MURF)-1 and Atrogin-1).

Rehabilitation improved peak work-rate and the 6-min walk distance similarly in non-cachectic (18 $\pm 3 \%$ and $42 \pm 13 \mathrm{~m}$, respectively) and cachectic (16 $\pm 2 \%$ and $53 \pm 16 \mathrm{~m}$, respectively) patients, but quality of life only improved in non-cachectic COPD. Mean muscle fibre cross-sectional area increased in both groups, but significantly less in cachectic $(7 \pm 2 \%)$ than in non-cachectic $(11 \pm 2 \%)$ patients. Both groups equally decreased the proportion of type Ilb fibres and increased muscle capillary/fibre ratio. IGF-I mRNA expression increased in both groups, but IGF-I protein levels increased more in non-cachectic COPD. MyoD was upregulated, whereas myostatin was downregulated at the mRNA and protein level only in non-cachectic patients. Whilst rehabilitation had no effect on TNF- $\alpha$ expression, it decreased the activation of the transcription factor NF- $\kappa B$ in both groups by the same amount. Atrogin-1 and MURF-1 expression were increased in cachectic COPD, but it was decreased in non-cachectic patients.

Cachectic COPD patients partially retain the capacity for peripheral muscle remodelling in response to rehabilitation and are able to increase exercise capacity as much as those without cachexia, even if they exhibit both quantitative and qualitative differences in the type of muscle fibre remodelling in response to exercise training.

KEYWORDS: Cachexia, chronic obstructive pulmonary disease, muscle remodelling, rehabilitative exercise

achexia refers to severe weight loss with disproportionate muscle wasting. Muscle wasting is common in many chronic diseases including chronic obstructive pulmonary disease (COPD) [1]. In COPD, muscle wasting (defined as a fat-free mass index (FFMI) $<16 \mathrm{~kg} \cdot \mathrm{m}^{-2}$ in males and $<15 \mathrm{~kg} \cdot \mathrm{m}^{-2}$ in females) occurs in about $20-40 \%$ of the patient population and is highly predictive of increased mortality [2]. The causes of cachexia in COPD are probably multiple, but remain to be established. Possibili- ties include energy imbalance, systemic inflammation, oxidative stress, hormonal insufficiency and arterial hypoxaemia, as well as physical inactivity [1-3].

Rehabilitative exercise training is recommended in many diseases associated with skeletal muscle wasting as a therapeutic strategy that could potentially attenuate muscle loss and promote muscle growth [4]. It has previously shown that in noncachectic patients with COPD, exercise training

This article has supplementary material accessible from www.erj.ersjournals.com

AFFILIATIONS

*Evangelismos Hospital, National and Kapodistrian University of Athens, Dept of Critical Care Medicine and Pulmonary Services Pulmonary Rehabilitation Centre, \#" "M. Simou, and G.P. Livanos Laboratories", National and Kapodistrian University of Athens, Dept of Critical Care Medicine and Pulmonary Services

"Depts of Physical Education and Sport Sciences, National and Kapodistrian University of Athens, and

${ }^{+}$Dept of Neurology, Eginition Hospital, National and Kapodistrian University of Athens, Athens, Greece. ${ }^{\S}$ Dept of Medicine, University of California San Diego, La Jolla, CA, USA.

CORRESPONDENCE

I. Vogiatzis

Thorax Foundation

3 Ploutarhou Str. 10675

Kolonaki

Athens

Greece

E-mail: gianvog@phed.uoa.gr

Received:

July 162009

Accepted after revision:

Jan 152010

First published online:

Jan 282010 
induces significant adaptations in peripheral muscle fibre size and typology that, in the absence of a decrease in systemic or local muscle inflammation, are accompanied by the upregulation of key factors governing skeletal muscle hypertrophy and regeneration (namely local muscle expression of insulin-like growth factor (IGF)-I and myogenic differentiation factor D (MyoD)) [5]. Whether skeletal muscle wasting can be mitigated by pulmonary rehabilitation in cachectic patients with COPD currently remains unknown. Several studies have shown that cachectic COPD patients have increased activity of their protein breakdown pathways, in particular the nuclear factor (NF)- $\kappa B$ activated ubiquitin/proteasomal pathway, leading to muscle loss $[6,7]$. Inflammatory cytokines, such as tumour necrosis factor (TNF) $-\alpha$, can activate the transcription factor NF- $\kappa$ B by ubiquitination and degradation of the inhibitory IкB family member [8]. The interaction of IкB masks the nuclear localisation sequence of the NF- $\kappa \mathrm{B}$ complex, thereby preventing nuclear translocation and maintaining NF- $\kappa B$ in an inactive state in the cytoplasmic compartment [9, 10]. Although the results of studies $[11,12]$ exploring the expression of local muscle TNF- $\alpha$ in patients with severe COPD are inconsistent, experimental data suggest that TNF- $\alpha$ can interfere with the hypertrophic action of muscle IGF-I [13] and inhibit myogenic differentiation by destabilising MyoD protein [14].

Furthermore, excess levels of myostatin (a strong negative regulator of muscle growth) have also been proposed as another potential mediator of muscle wasting, since myostatin inhibits myogenesis by downregulating MyoD expression [15].

It addition, it has recently been demonstrated that in COPD patients with muscle wasting, exercise training causes upregulation of inducible nitric oxide synthase through the activation of NF- $\mathrm{B}$ transcription factor, thereby inducing greater degrees of tyrosine nitration of quadriceps proteins [16] compared to COPD patients without muscle wasting. Increased protein nitrotyrosination may favour protein degradation through the ubiquitin proteasome pathway; therefore, limiting the capacity for muscle remodelling in patients with muscle cachexia. Besides cachexia, the NF- $\kappa B$ transcription factor complex has also been implicated in muscle atrophy attributable to muscle disuse and physical inactivity [17].

Based on the evidence above it was hypothesised that cachectic patients would respond poorly to pulmonary rehabilitation in terms of muscle fibre remodelling. Accordingly, we investigated the effects of pulmonary rehabilitation on peripheral muscle fibre morphological characteristics and on the local muscle expression of a number of factors that are known [5-7, 11-17] to govern the signalling pathways for muscle remodelling (i.e. IGF-I and its isoform mechano-growth factor (MGF), protein kinase B (Akt), MyoD, TNF- $\alpha$, I B, myostatin, Atrogin-1 and muscle ring finger protein (MURF)-1, as well as protein nitration levels) in cachectic patients with COPD. An additional purpose of our study was to compare the magnitude of rehabilitativeinduced peripheral muscle adaptations between cachectic and non-cachectic COPD patients.

\section{MATERIALS AND METHODS}

\section{Study population}

A total of 29 male patients with clinically stable COPD meeting the following criteria were recruited for this study: 1) post-bronchodilator forced expiratory volume in $1 \mathrm{~s}$ (FEV1) $<50 \%$ predicted and FEV1/forced vital capacity $<70 \%$ without significant post-bronchodilator reversibility $(<10 \%$ FEV1 \% pred normal); 2) optimal medical therapy without regular use of systemic corticosteroids; and 3) absence of other significant diseases that could contribute to exercise limitation. Of these, 10 patients were characterised by peripheral muscle atrophy (FFMI $<17 \mathrm{~kg} \cdot \mathrm{m}^{-2}$ [3]) as assessed by bioelectrical impedance (Bodystat 1500; Bodysat Ltd, Isle of Man, UK). Patients signed an informed consent that was approved by the National and Kapodistrian University of Athens, Eugenidion Hospital (Athens, Greece) ethics committee.

\section{Study design}

Both cachectic and non-cachectic patient groups were admitted to a multidisciplinary pulmonary rehabilitation programme as previously described [18] and detailed in the supplementary data. Prior to and upon completion of the programme, patients were assessed for pulmonary function, exercise tolerance and quality of life. A percutaneous muscle biopsy was also performed on all patients before and after rehabilitation.

\section{Assessment}

Initial assessment included: 1) resting pulmonary function and subdivisions of lung volumes by body plethysmography (Medgraphics Autolink 1085D; Medical Graphics, St Paul, MN, USA); 2) incremental cycle ergometer test (Ergoline 800; Sensor Medics Inc., Yorba Linda, CA, USA); 3) the 6-min walk test; and 4) the St George's Respiratory Questionnaire for assessing quality of life.

\section{Muscle biopsy}

Vastus lateralis muscle percutaneous biopsies were obtained $24 \mathrm{~h}$ after the first (baseline) and $24 \mathrm{~h}$ after the last (post) training session as described by BERGSTROM [19]. Biopsies were analysed blindly for fibre type classification, fibre crosssectional area and capillary to fibre ratio as previously described $[5,19]$ and in the supplementary data.

\section{Quantitative real-time PCR}

Total RNA was extracted from $30 \mathrm{mg}$ of muscle biopsies using RNeasy fibrous tissue (Qiagen, Crawley, UK). Quantitative real-time PCR was performed using a Chromo4 detector and a PTC-200 Peltier Thermal Cycler (both Bio Rad Life Sciences, Hercules, CA, USA) and analysed using the Opticon software 2.03 (MJ Research, Waltham, MA, USA). Primer sequences for TNF- $\alpha$, IGF-I, MGF (that is the load sensitive splice variant of IGF-I), MyoD, Akt, myostatin and the primer sequences for cloning partial sequences of each gene are given in the supplementary data (tables 1 and 2).

\section{Muscle protein immunoblotting}

Proteins were extracted using 10 volumes of a lysis buffer containing protease inhibitors (Complete Mini; Roche Diagnostics, Mannheim, Germany) and phosphatase inhibitors (Complete PhosSTOP; Roche Diagnostics). Western blotting techniques for measuring TNF- $\alpha$, IGF-I, MyoD, myostatin, Atrogin-1, MURF-1 and protein tyrosine nitration, as well as total and phosphorylated Akt and IкB proteins, together with the antibodies and dilutions are detailed in the supplementary data. 


\begin{tabular}{|c|c|c|c|}
\hline \multirow[t]{2}{*}{ TABLE 1} & \multicolumn{3}{|c|}{$\begin{array}{l}\text { Demographic, lung function, exercise tolerance } \\
\text { and quality of life assessment of non-cachectic } \\
\text { and cachectic chronic obstructive pulmonary } \\
\text { disease patients }\end{array}$} \\
\hline & & Non-cachectic & Cachectic \\
\hline \multicolumn{2}{|l|}{ Subjects $n$} & 19 & 10 \\
\hline \multicolumn{2}{|l|}{ Age yrs } & $67 \pm 2$ & $63 \pm 2$ \\
\hline \multicolumn{2}{|l|}{ Weight kg } & $77 \pm 3$ & $60 \pm 3^{*}$ \\
\hline \multicolumn{2}{|l|}{ BMI $\mathbf{k g} \cdot \mathrm{m}^{-2}$} & $27.8 \pm 1.2$ & $21.5 \pm 0.7^{*}$ \\
\hline \multicolumn{2}{|c|}{$\mathrm{FFM}$ index $\mathbf{k g} \cdot \mathrm{cm}^{-2}$} & $19.0 \pm 0.4$ & $15.6 \pm 0.4^{*}$ \\
\hline \multicolumn{2}{|l|}{ FEV 1 L } & $1.18 \pm 0.12$ & $0.98 \pm 0.14$ \\
\hline \multicolumn{2}{|l|}{ FEV $1 \%$ pred } & $44.1 \pm 4.8$ & $37.5 \pm 6.1$ \\
\hline \multicolumn{2}{|l|}{ FVC L } & $2.84 \pm 0.19$ & $2.49 \pm 0.17$ \\
\hline \multicolumn{2}{|l|}{ FVC \% pred } & $80.0 \pm 5.2$ & $72.9 \pm 6.0$ \\
\hline \multicolumn{2}{|l|}{ TLC \% pred } & $108.7 \pm 5.5$ & $126.2 \pm 7.9$ \\
\hline \multicolumn{2}{|l|}{ FRC $\%$ pred } & $152.3 \pm 10.5$ & $172.6 \pm 12.4$ \\
\hline \multicolumn{2}{|l|}{ RV \% pred } & $147.6 \pm 21.1$ & $178.7 \pm 40.8$ \\
\hline \multicolumn{2}{|l|}{$T L, C O \%$ pred } & $51.3 \pm 5.6$ & $42.8 \pm 4.9^{\star}$ \\
\hline \multicolumn{2}{|l|}{ Peak WR W } & $53 \pm 4$ & $43 \pm 6^{*}$ \\
\hline \multicolumn{2}{|c|}{ Peak $V^{\prime} \mathrm{O}_{2} \mathrm{~mL} \cdot \mathrm{kg}^{-1} \cdot \mathrm{min}^{-1}$} & $15 \pm 0.97$ & $14.1 \pm 1.62$ \\
\hline \multicolumn{2}{|c|}{ 6-min walking distance $\mathrm{m}$} & $344 \pm 21$ & $273 \pm 44^{*}$ \\
\hline \multicolumn{2}{|c|}{ SGRQ total score } & $45.22 \pm 3.71$ & $40.97 \pm 4.99^{\star}$ \\
\hline \multicolumn{4}{|c|}{$\begin{array}{l}\text { Data are presented as mean } \pm \text { SEM, unless otherwise stated. BMI: body mass } \\
\text { index; FFM: fat-free mass; FEV1: forced expiratory volume in } 1 \mathrm{~S} ; \% \text { pred: \% } \\
\text { predicted; FVC: forced vital capacity; TLC: total lung capacity; FRC: functional } \\
\text { residual capacity; RV: residual volume; } T \mathrm{~L}, \mathrm{CO} \text { : transfer factor of the lung for } \\
\text { carbon monoxide; WR: work rate; } V^{\prime} \mathrm{O}_{2} \text { : oxygen uptake; SGRQ: St George'S } \\
\text { Respiratory Questionnaire. }{ }^{*}: \mathrm{p}<0.05 \text { compared with non-cachectic patients } \\
\text { (unpaired t-test). }\end{array}$} \\
\hline
\end{tabular}

\section{Statistical analyses}

The main outcome measures were the mean muscle fibre cross sectional area, and the local muscle MGF and MyoD mRNA expression in cachectic patients. The minimum sample size for the group of cachectic patients was calculated based on $80 \%$ power and a two-sided 0.05 significance level using the Statistica 8.0 program (Statsoft Inc., Tulsa, OK, USA). A sample size capable of detecting a change following rehabilitation of $500 \mu \mathrm{m}^{2}$ in mean fibre cross sectional area, 0.6 units of MGF/ glyceraldehyde-3-phosphate dehydrogenase (GAPDH) mRNA expression and 1.0 unit of MyoD/GAPDH mRNA expression was estimated using data obtained from a previous study [5] and the following standard deviations: $980 \mu \mathrm{m}^{2}, 0.61$ and 0.85 units, respectively. The critical sample size was estimated to be 10 patients.

Data are presented as mean \pm SEM. SEM was chosen rather than SD because we were interested in the variance of the mean values rather than the inter-subject variance. Baseline demographics and muscle fibre morphological data between noncachectic and cachectic patients were compared by unpaired t-tests. Differences in protein expression between patient groups at baseline were tested by an unpaired one-sample t-tests after setting the mean baseline value of non-cachectic patients at $100 \%$. For muscle fibre morphological characteristics and for mRNA expression, pre- and post-training group comparisons were made by two-way ANOVA with repeated

\begin{tabular}{|c|c|c|c|}
\hline \multirow[t]{2}{*}{ TABLE 2} & \multicolumn{3}{|c|}{$\begin{array}{l}\text { Muscle fibre morphological characteristics of } \\
\text { non-cachectic and cachectic chronic obstructive } \\
\text { pulmonary disease patients at baseline }\end{array}$} \\
\hline & & Non-cachectic & Cachectic \\
\hline \multicolumn{2}{|c|}{ Subjects $\mathrm{n}$} & 19 & 10 \\
\hline \multicolumn{4}{|c|}{ Muscle fibre \% } \\
\hline \multicolumn{2}{|l|}{ Type I } & $32.0 \pm 3.2$ & $33.6 \pm 2.9$ \\
\hline \multicolumn{2}{|l|}{ Type ॥ } & $67.5 \pm 3.3$ & $65.9 \pm 3.0$ \\
\hline \multicolumn{2}{|l|}{ Type lla } & $52.3 \pm 3.7$ & $50.4 \pm 6.5$ \\
\hline \multicolumn{2}{|l|}{ Type IIb } & $15.2 \pm 2.2$ & $15.5 \pm 3.6$ \\
\hline \multicolumn{2}{|c|}{ Mean muscle fibre cross sectional area } & $4509 \pm 198$ & $3872 \pm 258^{*}$ \\
\hline \multicolumn{4}{|c|}{ Cross sectional area $\mu \mathrm{m}^{2}$} \\
\hline \multicolumn{2}{|l|}{ Type I } & $4716 \pm 271$ & $4717 \pm 215$ \\
\hline \multicolumn{2}{|l|}{ Type lla } & $4507 \pm 247$ & $3695 \pm 337^{*}$ \\
\hline \multicolumn{2}{|l|}{ Type IIb } & $3649 \pm 208$ & $2872 \pm 250^{*}$ \\
\hline \multicolumn{2}{|c|}{ Capillary/fibre ratio } & $1.41 \pm 0.10$ & $1.44 \pm 0.10$ \\
\hline
\end{tabular}

Data are presented as mean \pm SEM, unless otherwise stated. *: $p<0.05$ compared with non-cachectic at (unpaired t-test)

measures followed by the least square difference test for post hoc analyses. Rehabilitation-induced changes in protein expression within each group (presented relative to the mean baseline value set at $100 \%$ ) were carried out by one-sample $\mathrm{t}$ tests. Between-group comparisons of rehabilitation-induced changes in protein expression were made using unpaired $t$ tests. The level of statistical significance was set at $\mathrm{p}<0.05$.

\section{RESULTS}

\section{Baseline patient characteristics}

Cachectic and non-cachectic patients were well matched according to age and severity of airflow obstruction (table 1). However, cycle ergometer exercise tolerance, the 6-min walking distance and the total score of the St George's Respiratory Questionnaire were all lower $(p<0.05)$ in the cachectic patients (table 1). Vastus lateralis muscle fibre type distribution was not different between groups (table 2). In contrast, the mean muscle fibre cross-sectional area was lower $(p=0.035)$ in the cachectic patients (table 2). This difference was attributable to the lower $(\mathrm{p}<0.05)$ cross-sectional areas of type IIa and IIb fibres (table 2). Capillary/fibre ratio was not significantly different between groups (table 2).

\section{Effects of training on exercise tolerance and quality of life}

Mean training intensity sustained during the rehabilitation programme was not different between cachectic and noncachectic patients $(126 \pm 13 \%$ and $117 \pm 10 \%$ of baseline peak work-rate, respectively). Accordingly, peak work-rate on the cycle ergometer was increased by a similar percentage in both cachectic patients (by $16 \pm 2 \% ; \mathrm{p}=0.011$ ) and non-cachectic patients (by $18 \pm 3 \% ; p=0.001$ ). Similarly, the 6-min walk distance increased $(p<0.05)$ in both cachectic (by $53 \pm 16 \mathrm{~m}$ ) and non-cachectic patients (by $42 \pm 13 \mathrm{~m}$ ) with no difference between groups. However, none of the groups exhibited a clinically meaningful mean improvement $(>54 \mathrm{~m})$. The total St George's Respiratory Questionnaire score was improved (reduced) by a clinically meaningful margin ( $-6 \pm 2$ units) in 


\begin{tabular}{|c|c|c|c|c|c|c|c|}
\hline \multirow[t]{3}{*}{ TABLE 3} & \multicolumn{7}{|c|}{$\begin{array}{l}\text { Effect of rehabilitation on muscle fibre morphological characteristics and on the mRNA expression of anabolic and } \\
\text { catabolic factors }\end{array}$} \\
\hline & & \multicolumn{3}{|c|}{ Non-cachectic ${ }^{\#}$} & \multicolumn{3}{|c|}{ Cachectic } \\
\hline & & Pre & Post & $\%$ change & Pre & Post & $\%$ change \\
\hline \multicolumn{8}{|c|}{ Muscle fibre \% } \\
\hline Type I & & $32.0 \pm 3.2$ & $38.7 \pm 3.5^{+}$ & $6.6 \pm 2.3$ & $33.6 \pm 2.9$ & $30.0 \pm 6.6$ & $-3.4 \pm 3.6^{*}$ \\
\hline Type Ila & & $52.3 \pm 3.7$ & $51.1 \pm 3.9^{+}$ & $-1.1 \pm 2.3$ & $50.4 \pm 6.5$ & $58.0 \pm 5.3^{+}$ & $9.6 \pm 2.8^{*}$ \\
\hline Type IIb & & $15.2 \pm 2.2$ & $9.3 \pm 1.4^{+}$ & $-5.9 \pm 1.3$ & $15.5 \pm 3.6$ & $11.9 \pm 3.6^{+}$ & $-5.4 \pm 2.1$ \\
\hline Mean fibre & oss sectional area & $4509 \pm 198$ & $4947 \pm 227^{+}$ & $10.9 \pm 1.7$ & $3872 \pm 258^{*}$ & $4136 \pm 296^{+}$ & $7.3 \pm 1.7$ \\
\hline \multicolumn{8}{|c|}{ Cross sectional area $\mu \mathrm{m}^{2}$} \\
\hline Type I & & $4716 \pm 271$ & $5398 \pm 315^{+}$ & $16.4 \pm 4.8$ & $4717 \pm 215$ & $4796 \pm 152$ & $3.1 \pm 4.5^{\star}$ \\
\hline Type Ila & & $4507 \pm 247$ & $4954 \pm 251$ & $11.4 \pm 3.5$ & $3695 \pm 337^{*}$ & $4091 \pm 377^{+}$ & $12.1 \pm 3.2$ \\
\hline Type Ilb & & $3649 \pm 208$ & $3976 \pm 176^{+}$ & $11.6 \pm 4.3$ & $2872 \pm 250^{*}$ & $3224 \pm 388^{+}$ & $12.1 \pm 3.9$ \\
\hline Capillary/fib & e ratio & $1.41 \pm 0.1$ & $1.70 \pm 0.1^{+}$ & $20.3 \pm 4.1$ & $1.44 \pm 0.1$ & $1.61 \pm 0.1^{+}$ & $12.1 \pm 3.4$ \\
\hline mRNA IGF- & GAPDH expression & $0.72 \pm 0.11$ & $1.29 \pm 0.14^{+}$ & $79 \pm 3$ & $0.41 \pm 0.09$ & $0.77 \pm 0.10^{+}$ & $88 \pm 11$ \\
\hline mRNA MGF & APDH expression & $0.82 \pm 0.14$ & $1.21 \pm 0.13^{+}$ & $47 \pm 10$ & $1.18 \pm 0.31$ & $2.24 \pm 0.71^{+}$ & $90 \pm 29^{*}$ \\
\hline mRNA Myo & GAPDH expression & $1.30 \pm 2.83$ & $2.20 \pm 4.16^{+}$ & $69 \pm 17$ & $3.10 \pm 1.1$ & $2.20 \pm 8.7$ & $-29 \pm 6^{*}$ \\
\hline mRNA Myo & /GAPDH expression & $2.37 \pm 0.48$ & $1.78 \pm 0.44^{+}$ & $-25 \pm 11$ & $2.02 \pm 0.48$ & $1.96 \pm 0.27$ & $-3 \pm 4^{\star}$ \\
\hline mRNA TNF- & GAPDH expression & $0.91 \pm 0.27$ & $0.62 \pm 0.17$ & $-32 \pm 37$ & $1.52 \pm 0.42$ & $1.45 \pm 0.55^{+}$ & $-5 \pm 31^{*}$ \\
\hline
\end{tabular}

Data are presented as mean \pm SEM. All mRNA data were normalised against glyceraldehyde-3-phosphate dehydrogenase (GAPDH) number of copies, which were neither significantly different nor affected by exercise training in either of the two groups. IGF-I: insulin-like growth factor-l; MGF: mechano-growth factor; MyoD: myogenic differentiation factor D; Myost: myostatin; TNF- $\alpha$ : tumour necrosis factor- $\alpha{ }^{*}: n=19 ;{ }^{*}: n=10$. *: $p<0.05$ compared to non-cachectic; ${ }^{+}: p<0.05$ within-group differences.

non-cachectic patients only, whereas it remained unchanged $(-1 \pm 1$ units $)$ in cachectic patients.

\section{Vastus lateralis muscle fibre morphological adaptations}

Following rehabilitation, vastus lateralis mean muscle fibre cross-sectional area was increased in both cachectic (by $264 \pm$ $66 \mu^{2} ; \mathrm{p}=0.003$ ) and non-cachectic patients (by $438 \pm 66 \mu \mathrm{m} 2$; $\mathrm{p}<0.001)$; however, the increase was relatively greater $(\mathrm{p}=0.039)$ in the non-cachectic $(11 \pm 2 \%)$ compared to the cachectic patients $(7 \pm 2 \%)$ (table 3$)$. Although both groups increased cross-sectional areas of type IIa and IIb muscle fibres by a similar magnitude $(\mathrm{p}<0.01)$, only non-cachectic patients increased the cross-sectional area of type I fibres $(p=0.001)$ (table 3). In addition, whilst both groups equally decreased $(\mathrm{p}<0.005)$ the proportion of type Ilb fibres, non-cachectic patients increased the proportion of type I fibres $(p=0.01)$, whereas cachectic patients increased the proportion of type IIa fibres $(p=0.012)$ (table 3). Furthermore, capillary/fibre ratio was increased $(p<0.02)$ similarly in both groups (table 3$)$.

\section{Rehabilitation-induced molecular adaptations in peripheral muscle}

Western blotting revealed that at baseline, cachectic and noncachectic COPD patients exhibited different levels of expression among the remodelling markers tested (fig. 1a). Accordingly, cachectic patients showed significantly lower levels of expression of both IGF-I and MyoD. In contrast, the expression of TNF- $\alpha$, total I $\kappa$ B- $\alpha$ and myostatin was similar between groups (fig. 1a). Total Akt protein expression was significantly greater in the cachectic patients (fig. 1a). The ratio of phosphorylated I $\kappa \mathrm{B}-\alpha$ to total I $\kappa \mathrm{B}-\alpha$ was higher in the cachectic group (fig. $1 \mathrm{~b})$. In addition, at baseline the ratio of phosphorylated Akt to total Akt was also significantly greater in cachectic patients (fig. 1c). Prior to the initiation of the pulmonary rehabilitation programme there were no significant differences in protein expression of total tyrosine nitration and MURF-1 between cachectic and noncachectic patients. However, in the muscle of cachectic COPD patients, Atrogin-1 protein expression was significantly increased (by $145 \pm 54 \%$ ) compared to non-cachectic patients.

Post-training, there was an increase $(p<0.02)$ in the mRNA levels of IGF-I in both groups (table 3). Similarly, there was an increase $(\mathrm{p}<0.05)$ in the mRNA levels of MGF in both cachectic (from $1.18 \pm 0.31$ to $2.24 \pm 0.71 \mathrm{mRNA}$ copies $\cdot 10^{4} \mathrm{GAPDH}$ copies $^{-1}$ ) and non-cachectic patients (from $0.82 \pm 0.14$ to $1.21 \pm 0.13$ mRNA copies $\cdot 10^{4}$ GAPDH copies $^{-1}$ ). The increase in mRNA levels of MGF was greater $(p=0.027)$ in cachectic patients. In these patients rehabilitation-induced changes in MGF mRNA expression were significantly correlated $(r=0.65$, $p=0.034$ ) with the respective changes in cycle ergometry peak work-rate. In non-cachectic patients rehabilitation significantly increased IGF-I protein expression two-fold (fig. 2b). In contrast, cachectic patients did not show increased IGF-I protein expression after the rehabilitation programme. In addition, the post-training IGF-I protein expression levels were significantly greater in non-cachectic than cachectic patients (fig. 2b).

Non-cachectic patients exhibited a significant increase in MyoD mRNA after training (table 3), consistent with the percentage increase in protein levels (fig. 3c). However, cachectic patients did not show increased post-training MyoD mRNA (table 3) or protein (fig. 2c) expression. After training, the level of MyoD mRNA and protein expression in non-cachectic patients was significantly higher than in cachectic patients (fig. 2c). 


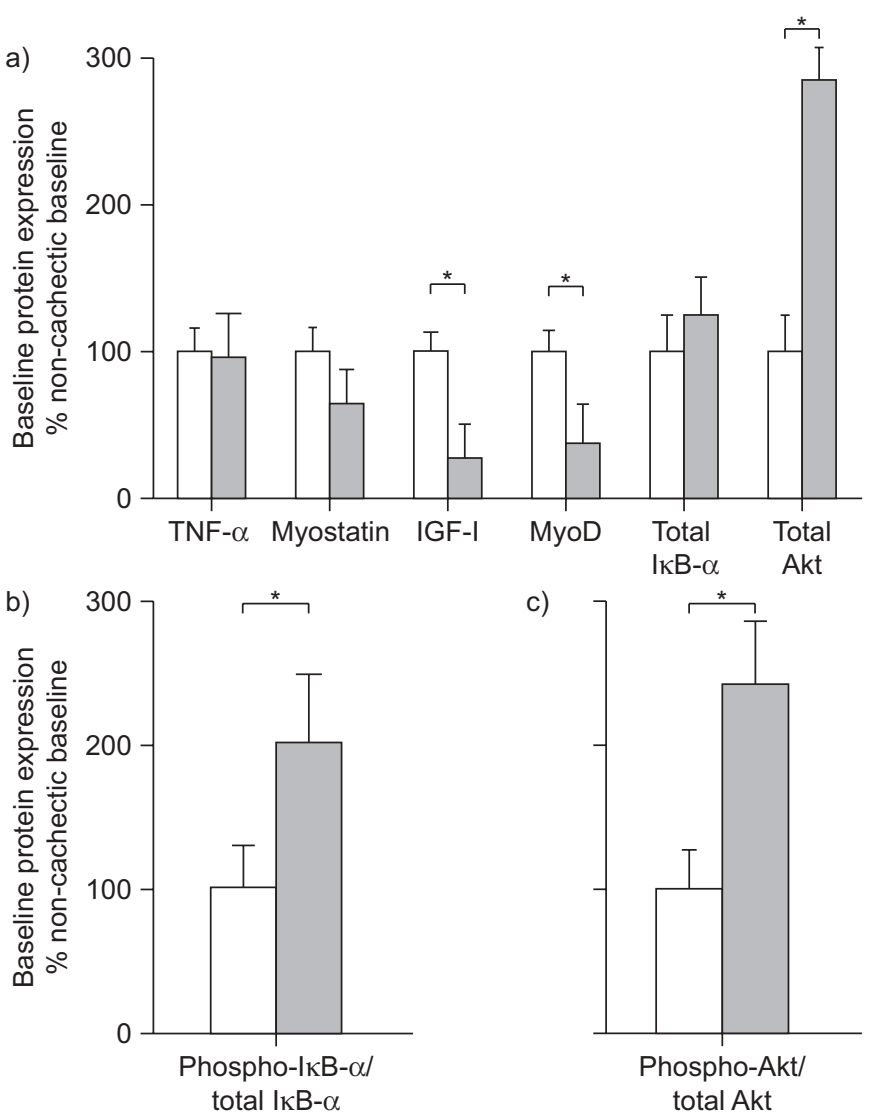

FIGURE 1. Factors associated with skeletal muscle hypertrophy and atrophy pathways at baseline. Protein expression of molecular markers in peripheral muscle homogenates from non-cachectic $(\square)$ and cachectic ( $\square$ ) chronic obstructive pulmonary disease patients were studied by western blot at baseline for a) the level of tumour necrosis factor (TNF)- $\alpha$, myostatin, insulin-like growth factor (IGF)-I, myogenic differentiation factor $\mathrm{D}(\mathrm{MyOD})$, total $\mid \kappa \mathrm{K}-\alpha$ and total Akt protein expression, b) the ratio of phosphorylated $\mid \kappa \mathrm{K}-\alpha /$ total $\mid \kappa \mathrm{K}-\alpha$, and $\mathrm{c}$ ) the ratio of phosphorylated Akt/total Akt. Results of densitometric analyses (mean \pm SEM) for cachectic patients are presented relative to the mean baseline values of noncachectic patients set at $100 \%$. *: $p<0.05$ between groups.

Rehabilitation significantly reduced myostatin mRNA (table 3) and protein levels (fig. 3b) in non-cachectic patients. In contrast, this was not seen in cachectic patients (fig. $3 b$ ). TNF- $\alpha$ was not affected at either mRNA (table 3) or protein levels (fig. 3c) by rehabilitative exercise in either group. Posttraining mRNA TNF- $\alpha$ expression was significantly greater in cachectic compared to non-cachectic patients (table 3).

Prior to rehabilitation, both groups of patients had the same amount of total cytoplasmic IкB- $\alpha$ (fig. 1a). After rehabilitation, the ratio of phosphorylated IкB- $\alpha$ to total IкB- $\alpha$ was significantly $(\mathrm{p}<0.05)$ decreased in both groups by the same amount (fig. $4 b)$. Furthermore, prior to rehabilitation, cachectic patients had greater amounts of total cytoplasmic Akt (fig. 1a). After rehabilitation, the ratio of phosphorylated Akt to total Akt did not increase in either cachectic or non-cachectic patients (fig. 4d).

Rehabilitation induced a significant increase in muscle protein nitration in cachectic patients only (fig. 5a). Interestingly, following exercise training, Atrogin-1 and MURF-1 protein a)

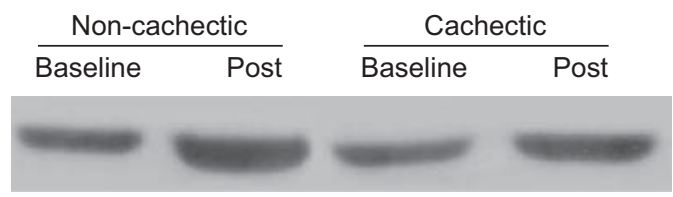

$\alpha$-Actinin

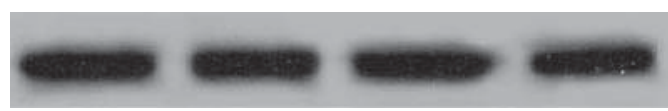

MyoD

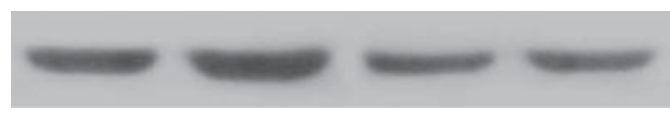

$\alpha-$-Actinin
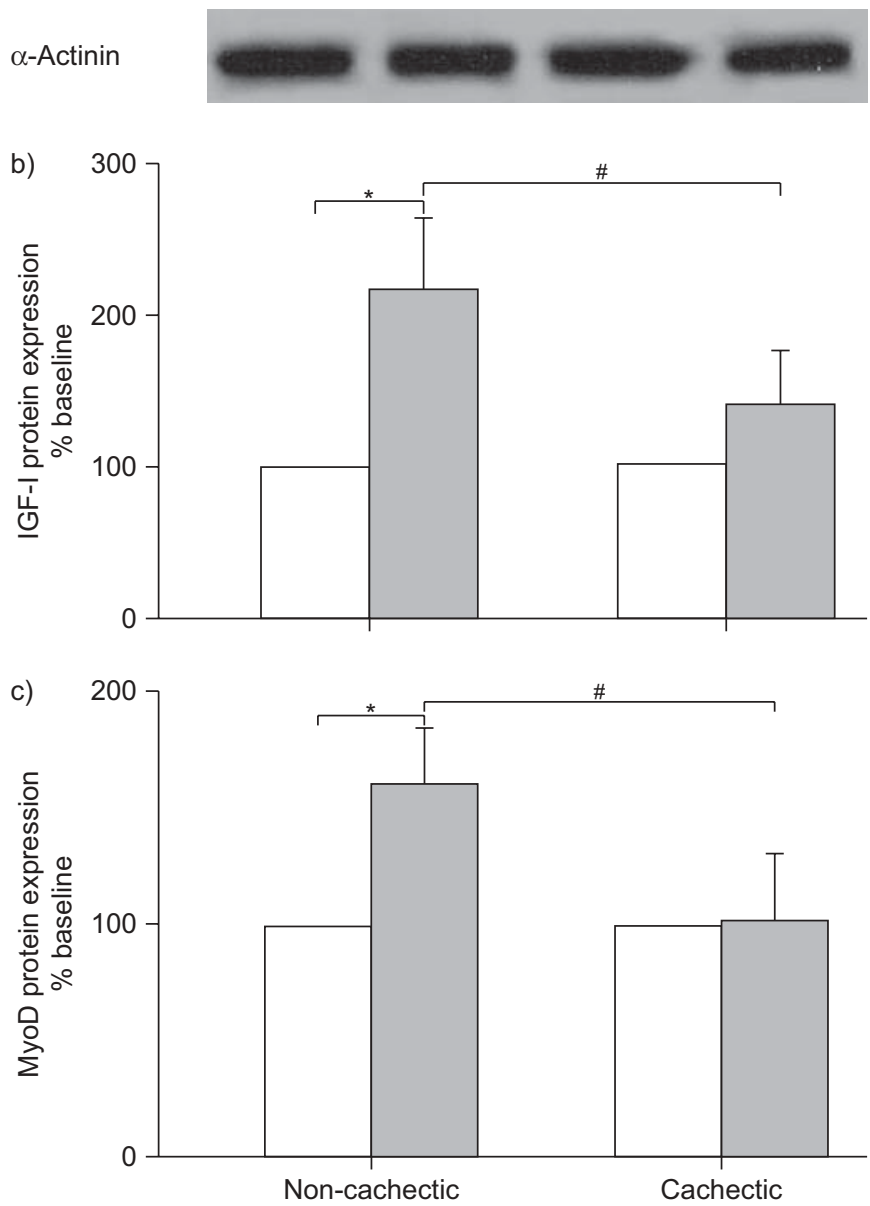

FIGURE 2. Insulin-like growth factor (IGF)-I and myogenic differentiation factor D (MyoD) expression following rehabilitation. a) Representative western blots for IGF-I and MyoD. b, c) Densitometric analyses of western blots from non-cachectic and cachectic chronic obstructive pulmonary disease patients for b) IGF-I and c) MyoD. Rehabilitation-induced changes in protein expression within each group are presented relative to the mean baseline value set at $100 \%$. Results are presented as mean \pm SEM. $\square$ : baseline; $\square$ : post-training. ${ }^{*}$ : at $p<0.05$ between baseline and post-rehabilitation for each group; ${ }^{*}$ : significant differences between groups.

expression was significantly increased in cachectic patients, whereas it was significantly decreased in non-cachectic patients (fig. 5c and d).

\section{DISCUSSION}

Our study shows that rehabilitative exercise training significantly improves exercise tolerance and induces significant 
a)

\begin{tabular}{|c|c|c|c|}
\hline \multicolumn{2}{|c|}{ Non-cachectic } & \multicolumn{2}{|c|}{ Cachectic } \\
\hline Baseline & Post & Baseline & Post \\
\hline
\end{tabular}

$\alpha-$ Actinin

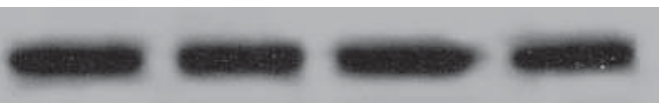

TNF- $\alpha$

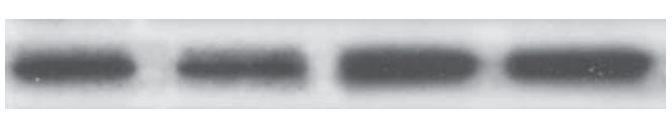

$\alpha$-Actinin
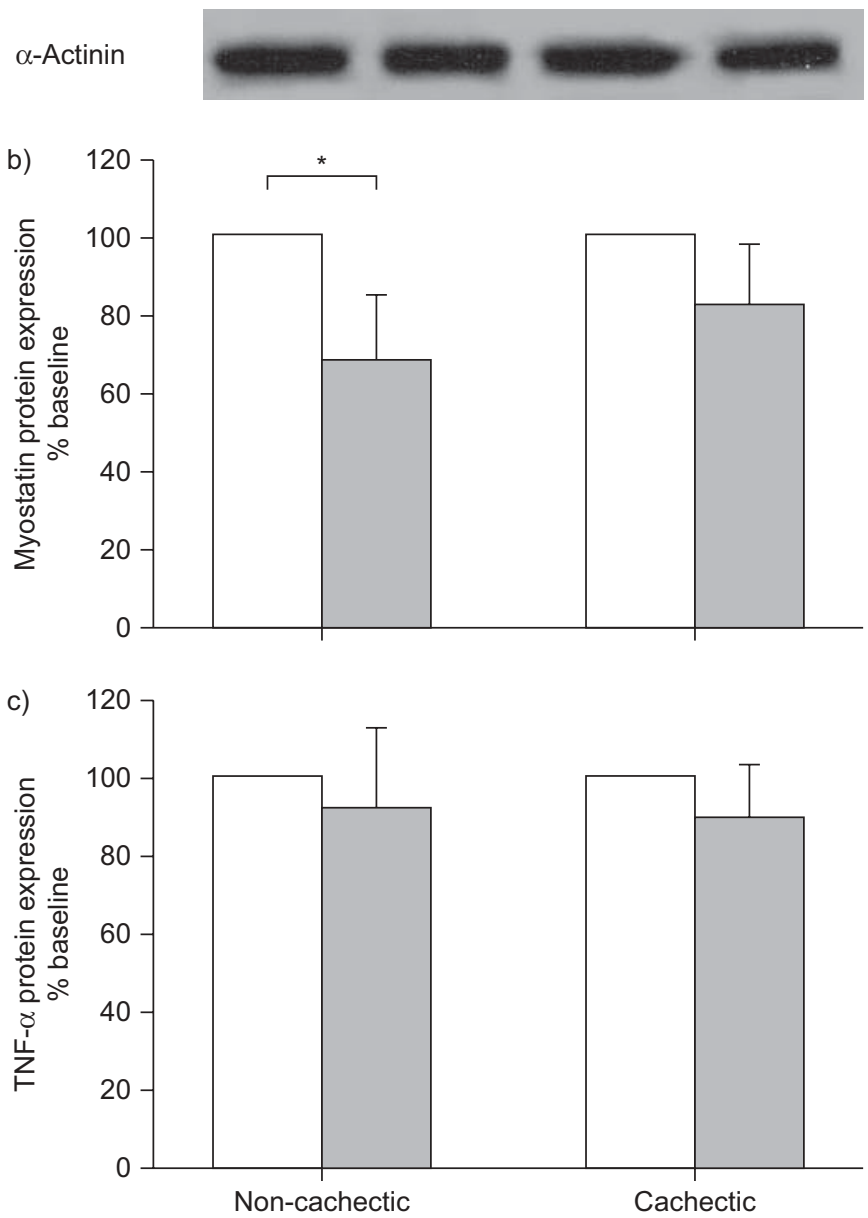

FIGURE 3. Myostatin and tumour necrosis factor (TNF)- $\alpha$ expression. a) Representative western blots for Myostatin and TNF- $\alpha$. b, c) Densitometric analyses of western blots from non-cachectic and cachectic chronic obstructive pulmonary disease patients for b) Myostatin and c) TNF- $\alpha$. Rehabilitation-induced changes in protein expression within each group are presented relative to the mean baseline value set at $100 \%$. Results are presented as mean \pm SEM. $\square$ : baseline; $\square$ : posttraining. *: $p<0.05$ between baseline and post-rehabilitation for each group.

adaptations in vastus lateralis muscle fibre size, typology and capillarisation in cachectic patients with COPD. Whilst in these patients rehabilitation had no effect on myostatin or TNF- $\alpha$ protein expression, it decreased the activation of the transcription factor NF- $\kappa B$ by the same amount as in non-cachectic patients. Furthermore, even if the magnitude of increase in the mean muscle fibre cross-sectional area and the proportion of type I muscle fibres was greater in non-cachectic patients with COPD, those with cachexia were able to increase exercise capacity as much as those without cachexia. The observed limited training effect on muscle fibre remodelling in cachectic patients could be attributed to simultaneous activation of hypertrophy (expression of local muscle growth factors) and atrophy (ubiquitin/proteasome) signalling pathways by exercise training. In non-cachectic patients, the type of muscle fibre remodelling in terms of expression of signalling pathways (primarily hypertophic) and of phenotypical adaptations were different among cachectic and non-cachectic COPD. Thus, it is apparent that exercise training produced both quantitative and qualitative differences in muscle fibre remodelling among patients populations.

\section{Baseline muscle remodelling markers}

The biochemical pathways engaged in the development of muscle atrophy are thought to result from an imbalance between protein synthesis and breakdown; however, the exact mechanisms involved are not fully understood [17]. Accordingly, molecular factors regulating muscle remodelling were analysed at baseline and compared between patient groups. Our study shows for the first time that baseline protein expression of Atrogin-1, a ligase regulating ubiquitin-mediated protein degradation [9], was significantly increased in the muscle of cachectic patients, whereas IGF-I and MyoD protein expression were lower in cachectic patients (fig. 1). Other studies have compared IGF-I and MyoD expression between COPD patients and healthy age-matched subjects [20] and found that the expression of these two anabolic markers were reduced in hospitalised and clinically stable COPD patients compared to healthy controls. A study by CRUL et al. [20] suggested that diminished physical activity may be the main mechanism accounting for decreased muscle IGF-I and MyoD levels in COPD. In support of this notion, physical inactivity has been shown to be associated with decreased IGF-I mRNA levels and alterations in the MyoD/myogenin expression ratio in a mouse muscle-disuse model $[21,22]$. In contrast to IGF-I and MyoD protein expression, we found that the phosphorylated Akt to total Akt ratio was significantly increased in cachectic COPD (fig. 1c), an indication previously interpreted as a failed attempt to maintain or restore muscle mass in patients with COPD [23].

However, greater muscle fibre atrophy in cachectic compared to non-cachectic patients could also be explained by greater activation of the NF- $\kappa \mathrm{B}$ transcription factor complex [6, 7]. Although at baseline cachectic and non-cachectic patients did not differ in terms of the level of expression of total I $\mathrm{I} B-\alpha$

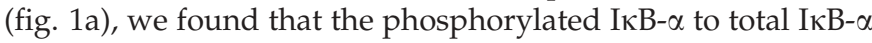
ratio was two-fold greater in cachectic compared to noncachectic patients (fig. 1b). However, interpretation of these findings has to be carried out with caution because the IкB- $\alpha$ ratio utilised is an indicator of the NF- $\mathrm{B}$ activation and, as such, further experiments measuring activation of NF- $\kappa B$ in the nucleus were not possible due to muscle tissue limitation.

NF- $\kappa \mathrm{B}$ can be activated by a number of cachexia-associated factors including TNF- $\alpha$ [7]. Although we did not find significant differences in baseline local muscle mRNA or protein TNF- $\alpha$ expression between study groups (fig. 1a), we can not exclude the possibility that the greater degree of muscle wasting seen in cachectic COPD was instigated by increased local muscle expression of cytokines (other than 
a)
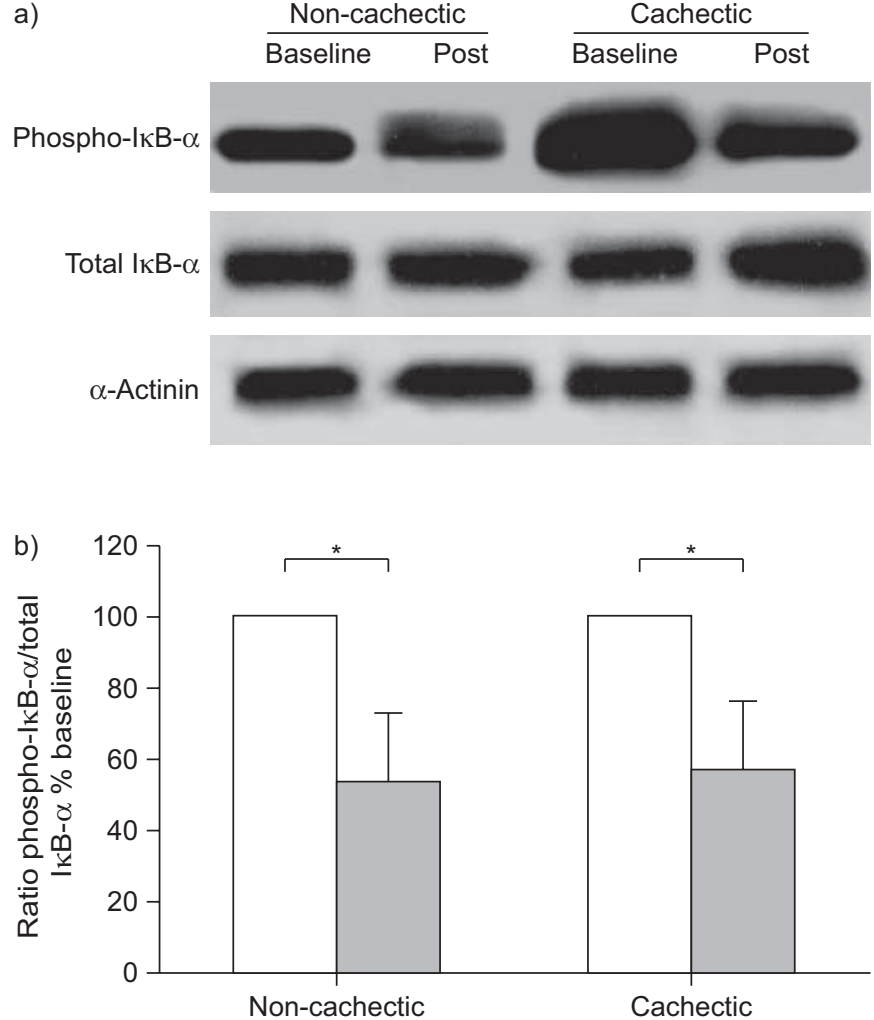

c)
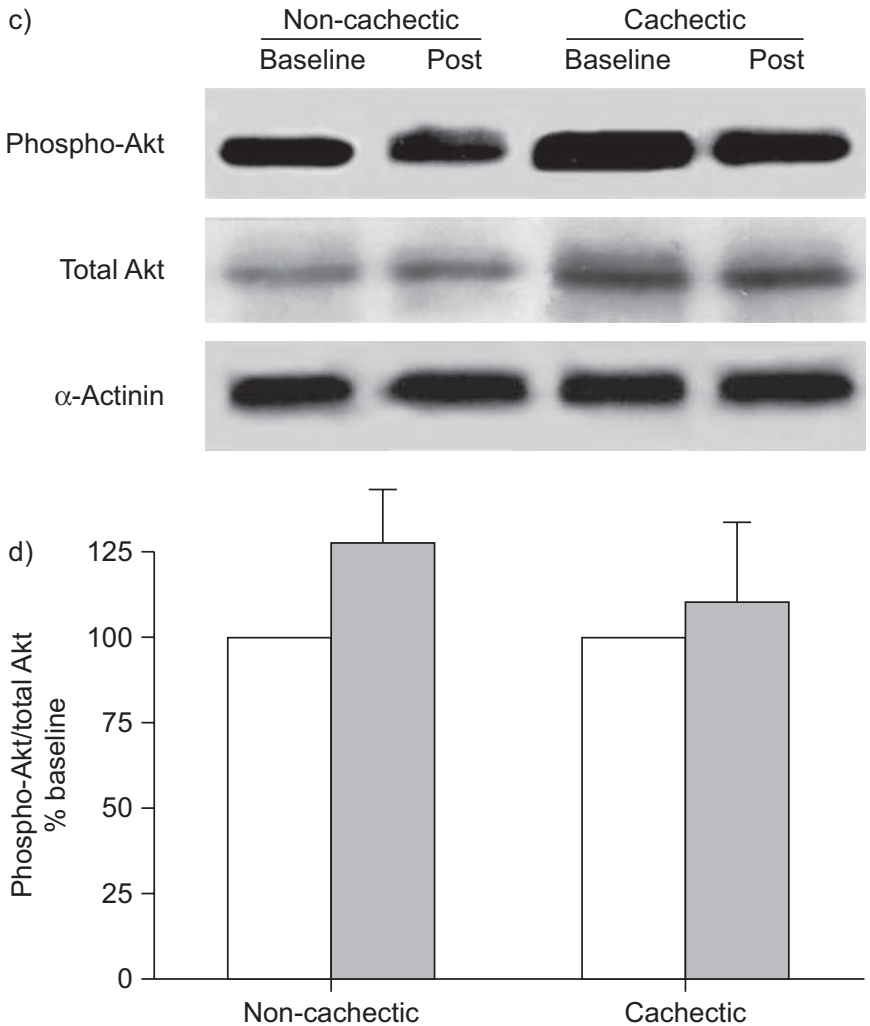

FIGURE 4. Pulmonary rehabilitation decreases nuclear factor (NF)-kB activation and tends to increase Akt activation. Representative western blots for a) phosphorylated I KB- $\alpha$, Total I $\mathrm{\kappa B}-\alpha$ and $\alpha$-Actinin and c) phosphorylated Akt, Total Akt and $\alpha$-Actinin. Densitometric analyses of blots studied in non-cachectic and cachectic chronic obstructive pulmonary disease patients for b) phosphorylated IkB- $\alpha$ and d) phosphorylated Akt. Rehabilitation-induced changes in protein expression within each group are presented relative to the mean baseline value set at $100 \%$. Results are presented as mean \pm SEM. $\square$ : baseline; $\square$ : post-training. ${ }^{*}$ : $p<0.05$ between baseline and postrehabilitation for each group.

TNF- $\alpha$ ) which are involved in inflammation, such as interleukin (IL)-1 $\beta$ and IL-6, and which are thought to trigger muscle wasting via NF- $\kappa$ B activation [24]. Nevertheless, a recent study [24] demonstrated muscle atrophy due to systemic inflammation-dependent chronic activation of NF$\kappa \mathrm{B}$ in the muscle of mice without increased TNF- $\alpha$, IL- 6 or IL- 8 mRNA levels. Consistent with a recent study [16], at baseline we found no differences in muscle protein tyrosine nitration between cachectic and non-cachectic patients.

In muscle, NF- $\kappa \mathrm{B}$ can also inhibit mRNA and protein expression of MyoD [14, 25], an essential transcription factor in myogenesis [9]. Downregulation of MyoD expression can also be induced by over-expression of myostatin, a strong negative regulator of muscle growth [15]. However, at baseline we found that myostatin protein expression was not different in cachectic patients compared to non-cachectic patients, thereby suggesting that the lower MyoD expression in cachectic patients is probably due to the increased activation of NF- $\mathrm{B}[6,7]$.

\section{Exercise-induced effects on exercise capacity and muscle remodelling markers}

Cachectic and non-cachectic COPD patients were selected to control for differences in patient phenotype. At the beginning of the study, cachectic patients were characterised by poorer exercise capacity and greater peripheral muscle fibre atrophy (tables 1 and 2). Following rehabilitation, exercise capacity was increased similarly in both cachectic and non-cachectic patients, most probably as a result of exercising both groups at a similar overall training load.

In addition, confirming previous findings in non-cachectic COPD [5], we have shown for the first time that rehabilitation induces a significant increase in vastus lateralis muscle mean fibre cross-sectional area and capillarisation in cachectic patients. In addition, rehabilitative exercise reduced the proportion of IIb fibres in favour of the more oxidative fibres (i.e. type IIa). These findings suggest that peripheral muscle remodelling in response to exercise training is, at least partially, preserved in cachectic patients with COPD. Indeed, we observed that muscle fibre hypertrophy was accompanied by significant upregulation of local muscle IGF-I and MGF mRNA expression that are known to play an important role in the hypertophic adaptation of muscle to overload [23, 26]. In our study the magnitude of increase in IGF-I mRNA expression after rehabilitation in cachectic COPD (by 88\%) was similar to that reported for the non-cachectic patients in the present (by 79\%) and previous (by 75\%) studies [5]. However, the increase in IGF-I mRNA expression after rehabilitation was translated at protein level in non-cachectic COPD only (fig. 2b); this may explain the greater degree of peripheral muscle fibre hypertrophy found in this group and highlights a potential post-transcriptional modification in cachectic 


\section{a)}

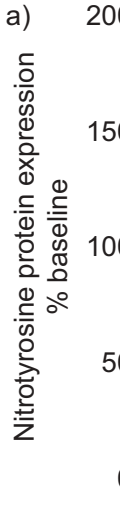

200

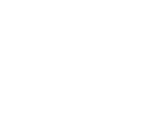

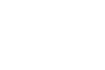

$\stackrel{*}{\square}$

(

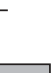

$150-$
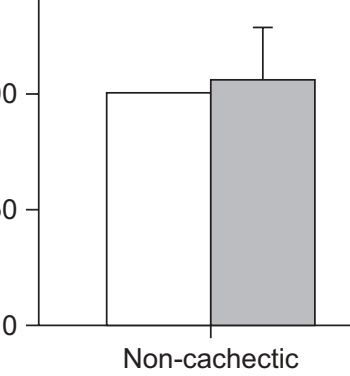

Non-cachectic

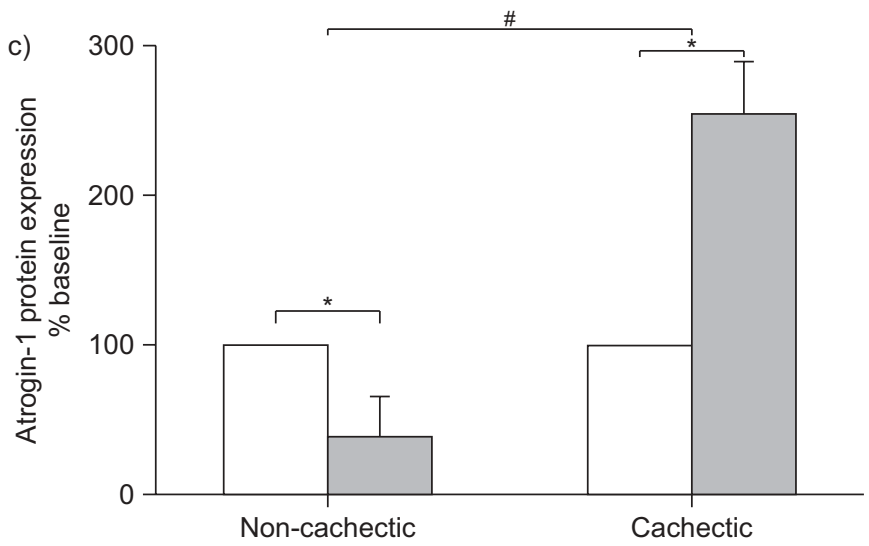

b)

Atrogin-1

MURF-1

$\alpha$-Actinin

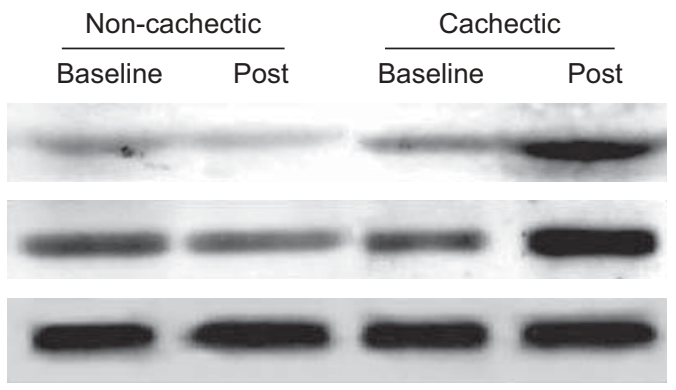

d)

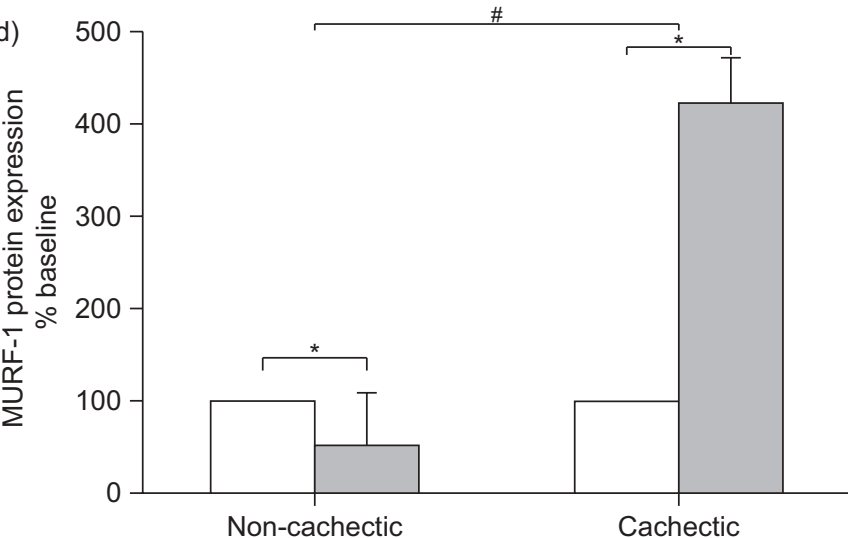

FIGURE 5. Pulmonary rehabilitation increases nitrotyrosine, Atrogin-1 and muscle ring finger protein (MURF)-1 protein expression in cachectic patients. a, c, d) Densitometric analyses of blots studied in non-cachectic and cachectic chronic obstructive pulmonary disease patients for a) protein tyrosine nitration, c) Atrogin-1 and d) MURF-1. b) Representative western blots for Atrogin-1, MURF-1 and $\alpha$-Actinin. Rehabilitation-induced changes in protein expression within each group are presented relative to the mean baseline value set at $100 \%$. Results are presented as mean \pm SEM. $\square$ : baseline; $\square$ : post-training. *: $p<0.05$ between baseline and post-rehabilitation for each group; \#: significant differences between groups.

patients. In addition, mRNA degradation may also act as an important mechanism in the regulation for the expression of muscle IGF-I [27].

Another potential factor that may account for the lower degree of peripheral muscle fibre hypertrophy observed in cachectic patients is their inability to upregulate local muscle MyoD in response to rehabilitative exercise (table 3, fig. 2c). MyoD is expressed in muscle satellite cells and mature myofibres and has been implicated in mediating the process of cell proliferation and differentiation for subsequent muscle regeneration and hypertrophy [14].

Within our study we investigated the effects of exercise training on two factors that are known to modulate the expression of local muscle MyoD; namely TNF- $\alpha$ and myostatin $[14,15]$. The downregulation of myostatin in response to exercise training in healthy humans has been shown to be essential for muscle growth [28]. In non-cachectic COPD patients, resistance training has been shown to induce a modest reduction in myostatin mRNA abundance [29]. Therefore, the results of the present study expand those in non-cachectic COPD [29] by demonstrating that endurance training induced significant reductions in both mRNA and protein myostatin expression (table 3, fig. 3b). Rehabilitationinduced reduction in local muscle myostatin expression in non-cachectic patients was accompanied by a significant upregulation of both mRNA and protein expression for MyoD, thereby confirming the results of a previous study [5]. In contrast, in cachectic patients neither mRNA nor protein expression for myostatin was significantly reduced (table 3, fig. 3b). This finding may explain the lack of significant training-induced upregulation of local muscle MyoD expression in cachectic COPD and account for the lower degree of fibre hypertrophy.

\section{Relationship between markers of inflammation and training responses}

Muscle TNF- $\alpha$ mRNA and protein expression were not significantly affected by exercise training in cachectic patients (table 3, fig. 3c), thus expanding the results of previous studies carried out in patients without peripheral muscle wasting [5, 30]. Interestingly, we found that the phosphorylated IкB- $\alpha$ to

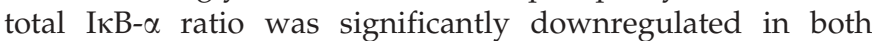
groups following rehabilitation, most probably indicating reduced activation of the NF- $\mathrm{KB}$ transcription factor (fig. $4 \mathrm{~b}$ ).

Emerging evidence suggests that NF- $\mathrm{KB}$ is involved in one of the most important signalling pathways linked to the loss of skeletal muscle mass in various physiological and pathophysiological conditions. A recent study using an animal model has provided unequivocal evidence that specific modulation of 
$\mathrm{NF}-\kappa \mathrm{B}$ activity can prevent skeletal muscle loss [31]. It has recently been recognised that NF- $\kappa B$ can be activated not only by cachectic factors (such as TNF- $\alpha$ ) but also by muscle disuse $[9,32]$. Muscle disuse triggers an NF- $\mathrm{B}$ activation pathway that is distinctly different from that triggered by a cytokine (e.g. TNF- $\alpha$ ) [32]. If this is correct, one would expect that exercise training would decrease the phosphorylation ratio of the inhibitory I $\kappa \mathrm{B}-\alpha$, which is in fact what our study demonstrates (fig. 4b), thereby indicating NF- $\kappa \mathrm{B}$ as a possible target for exercise training. Thus, it is possible that when disused, atrophic peripheral muscles of COPD patients undergo exercise training and NF- $\mathrm{\kappa B}$ is progressively de-activated, as indicated by the decreased phosphorylation of I $\mathrm{B}-\alpha$, allowing a number of downstream signalling pathways to be activated and promote muscle hypertrophy. Conversely, as baseline NF$\kappa \mathrm{B}$ activity was twice as high in cachectic patients compared to non-cachectic patients (fig. 1b), and since the phosphorylated I $\kappa-\alpha$ to total I $\kappa$ B $-\alpha$ ratio was downregulated in both groups following rehabilitation by the same magnitude (fig. $4 \mathrm{~b}$ ), it is conceivable that NF- $\kappa \mathrm{B}$ activity was still greater in cachectic patients following rehabilitation, thereby limiting the degree of muscle remodelling.

The limited degree of muscle remodelling in cachectic compared to non-cachectic patients can also be justified by our findings that exercise training caused a greater induction of muscle protein nitrotyrosination compared to non-cachectic patients [16], most likely facilitating degradation through the ubiquitin/proteasome pathway [6]. Our study is the first to provide proof for this notion since we found that two specific regulators of ubiquitin-mediated muscle proteolytic pathway (Atrogin-1 and MURF-1) were upregulated following rehabilitation in cachectic patients only. In contrast, in non-cachectic patients, Atrogin-1 and MURF-1 protein expression was downregulated, whereas growth and regeneration factors were unregulated; thus, justifying the greater degree of muscle fibre hypertrophy in these patients. The diverse response to exercise training between cachectic and non-cachectic patients resembles that recently documented in COPD patients and age-matched healthy controls where the protein degradation pathway was upregulated in COPD only [33]. Therefore, it is likely that exercise training predominantly produces an anabolic influence among non-cachectic COPD, while in cachectic COPD regular muscular activity simultaneously activates anabolic and catabolic pathways, thereby limiting the magnitude of the training effect.

\section{Study limitations}

The invasive nature of the muscle biopsies precluded a parallel, age-matched, healthy control training group which would have allowed comparisons in training-induced changes in local muscle anabolic and catabolic factors between healthy subjects and COPD patients with or without muscle wasting. In addition, for the same reason, a control COPD group was not studied, i.e. not undertaking rehabilitation. Such a group would have distinguished which of the effects of rehabilitation were due to a true effect of training and which are due to between-occasion muscle sampling differences. Furthermore, absence of quadriceps measurements of strength or mid-thigh cross-sectional area is acknowledged as a limitation in our study. However, recent studies $[23,34]$ have shown reduced mid-thigh cross-sectional area, as well as strength in cachectic patients compared to non-cachectic COPD patients and healthy controls. Furthermore, both in vivo and in vitro muscle function measurements would have provided more adequate evidence to link the observed molecular/cellular changes with the clinical outcomes of pulmonary rehabilitation in cachectic COPD. Another potential limitation in our study was that cachectic patients were also those showing worse lung function. Indeed, it is well known that weight loss and increasing severity of COPD are often associated with each other and both lead to poor prognosis of the disease [2].

In conclusion, although skeletal muscle training produces greater peripheral muscle fibre phenotypical adaptations in non-cachectic patients compared to cachectic patients with COPD, cachectic patients retain the capacity for peripheral muscle remodelling in response to rehabilitation and are able to increase exercise capacity as much as those without cachexia.

\section{SUPPORT STATEMENT}

This work was supported by the Thorax Foundation and by grants from the "A. Perotti" visiting Professorship fund of the Thorax Foundation. P.D. Wagner was supported, in part, by the National Institutes of Health (NIH R01 HL84281 and NIH P01 HL091830).

\section{STATEMENT OF INTEREST}

None declared.

\section{REFERENCES}

1 Morley JE, Thomas DR, Wilson M. Cachexia: pathophysiology and clinical relevance. Am J Clin Nutr 2006; 83: 735-743.

2 Schols AMWJ, Broekhuizen R, Weling-Scheepers CA, et al. Body composition and mortality in chronic obstructive pulmonary disease. Am J Clin Nutr 2005; 82: 53-59.

3 Wagner PD. Possible mechanisms underlying the development of cachexia in COPD. Eur Respir J 2008; 31: 492-501.

4 Zinna EM, Yarasheski KE. Exercise treatment to counteract protein wasting of chronic diseases. Curr Opin Clin Nutr Metab Care 2003; 6: 87-93.

5 Vogiatzis I, Stratakos G, Simoes DCM, et al. Effects of rehabilitative exercise on peripheral muscle TNF- $\alpha$, IL-6, IGF-I and MyoD expression in patients with COPD. Thorax 2007; 62: 950-956.

6 Agusti A, Morla M, Sauleda J, et al. NF- $\kappa$ B activation and iNOS upregulation in skeletal muscle of patients with COPD and low body weight. Thorax 2004; 59: 483-487.

7 Rutten EPA, Franssen FME, Engelen MPKJ, et al. Greater wholebody myofibrillar protein breakdown in cachectic patients with chronic obstructive pulmonary disease. Am J Clin Nutr 2006; 83: 829-834.

8 Yaron A, Hatzubai A, Davis M, et al. Identification of the receptor component of the IкB $\alpha$-ubiquitin ligase. Nature 1998; 396: 590-594

9 Jackman RW, Kandarian SC. The molecular basis of skeletal muscle atrophy. Am J Physiol Cell Physiol 2004; 287: C834-C843.

10 Li Y-P, Schwartz RJ, Waddell ID, et al. Skeletal muscle myocytes undergo protein loss and reactive oxygen-mediated NF- $\mathrm{B}$ activation in response to tumor necrosis factor- $\alpha$. FASEB J 1998; 12: $871-880$

11 Barreiro E, Schols AMWJ, Polkey MI, et al. Cytokine profile in quadriceps muscles of patients with severe COPD. Thorax 2008; 63 100-107.

12 Montes de Oca M, Torres SH, De Sanctis J, et al. Skeletal muscle inflammation and nitric oxide in patients with COPD. Eur Respir J 2005; 26: 390-397. 
13 Broussard SR, McCusker RH, Novakofski JE, et al. Cytokine-hormone interactions: tumor necrosis factor- $\alpha$ impairs biologic activity and downstream activation signals of the insulin-like growth factor I receptor in myoblasts. Endocrinology 2003; 144: 2988-2996.

14 Langen RCJ, Van Der Velden JLJ, Schols AMWJ, et al. Tumor necrosis factor-alpha inhibits myogenic differentiation through MyoD protein destabilization. FASEB J 2004; 18: 227-237.

15 Langley B, Thomas M, Bishop A, et al. Myostatin Inhibits myoblast differentiation by down-regulating MyoD expression. J Biol Chem 2002; 277: 49831-49840.

16 Barreiro E, Rabinovich R, Marin-Corral J, et al. Chronic endurance exercise induces quadriceps nitrosative stress in severe COPD patients. Thorax 2009; 64: 13-19.

17 Glass DJ. Skeletal muscle hypertrophy and atrophy signaling pathways. Int J Biochem Cell Biol 2005; 37: 1974-1984.

18 Vogiatzis I, Terzis G, Nanas S, et al. Skeletal muscle adaptations to interval training in patients with advanced COPD. Chest 2005; 128 : 3838-3845.

19 Bergstrom J. Percutaneous needle biopsy of skeletal muscle in physiological and clinical research. Scand J Clin Lab Invest 1975; 35 : 609-616.

20 Crul T, Spruit MA, Gayan-Ramirez G, et al. Markers of inflammation and disuse in vastus lateralis of chronic obstructive pulmonary disease patients. Eur J Clin Invest 2007; 37: 897-904.

21 Awede B, Thissen J, Gailly P, et al. Regulation of IGF-I, IGFBP-4 and IGFBP-5 gene expression by loading in mouse skeletal muscle. FEBS Lett 1999; 461: 263-267.

22 Mozdziak PE, Greaser ML, Schultz E. Myogenin, MyoD, and myosin heavy chain isoform expression following hindlimb suspension. Aviat Space Environ Med 1999; 70: 511-516.

23 Doucet M, Russell AP, Leger B, et al. Muscle atrophy and hypertrophy signaling in patients with chronic obstructive pulmonary disease. Am J Respir Crit Care Med 2007; 176: 261-269.
24 Cai D, Frantz JD, Tawa NE Jr, et al. IKK $\beta /$ NF- $\kappa$ B activation causes severe muscle wasting in mice. Cell 2004; 119: 285-298.

25 Di Marco S, Mazroui R, Dallaire P, et al. NF- $\kappa$ B-mediated MyoD decay during muscle wasting requires nitric oxide synthase mRNA stabilization, HuR protein, and nitric oxide release. Mol Cell Biol 2005; 25: 6533-6545.

26 Kostek MC, Delmonico MJ, Reichel JB, et al. Muscle strength response to strength training is influenced by insulin-like growth factor 1 genotype in older adults. J Appl Physiol 2005; 98: 2147-2154.

27 Moore MJ. From birth to death: the complex lives of eukaryotic mRNAs. Science 2005; 309: 1514-1518.

28 Jones SW, Hill RJ, Krasney PA, et al. Disuse atrophy and exercise rehabilitation in humans profoundly affects the expression of genes associated with the regulation of skeletal muscle mass. FASEB J 2004; 18: 1025-1037.

29 Lewis MI, Fournier M, Storer TW, et al. Skeletal muscle adaptations to testosterone and resistance training in men with COPD. J Appl Physiol 2007; 103: 1299-1310.

30 Langen RCJ, Schols AMWJ. Inflammation: friend or foe of muscle remodelling in COPD? Eur Respir J 2007; 30: 605-607.

31 Van Gammeren D, Damrauer JS, Jackman RW, et al. The IkappaB kinases IKKalpha and IKKbeta are necessary and sufficient for skeletal muscle atrophy. FASEB J 2009; 23: 362-370.

32 Hunter RB, Stevenson EJ, Koncarevic A, et al. Activation of an alternative NF- $\mathrm{KB}$ pathway in skeletal muscle during disuse atrophy. FASEB J 2002; 16: 529-538.

33 Radom-Aizik S, Kaminski N, Hayek S, et al. Effects of exercise training on quadriceps muscle gene expression in chronic obstructive pulmonary disease. J Appl Physiol 2007; 102: 1976-1984.

34 Plant PJ, Brooks D, Faughnan M, et al. Cellular markers of muscle atrophy in chronic obstructive pulmonary disease. Am J Respir Cell Mol Biol 2010; 42: 461-471. 